PROCEEDINGS OF THE

AMERICAN MATHEMATICAL SOCIETY

Volume 133, Number 4, Pages 1119-1126

S 0002-9939(04)07805-0

Article electronically published on October 18, 2004

\title{
ON QUESTIONS OF FATOU AND EREMENKO
}

\author{
P. J. RIPPON AND G. M. STALLARD
}

(Communicated by Michael Handel)

This paper is dedicated to the memory of Professor Noel Baker

\begin{abstract}
Let $f$ be a transcendental entire function and let $I(f)$ be the set of points whose iterates under $f$ tend to infinity. We show that $I(f)$ has at least one unbounded component. In the case that $f$ has a Baker wandering domain, we show that $I(f)$ is a connected unbounded set.
\end{abstract}

\section{INTRODUCTION}

Let $f$ be a transcendental entire function and denote by $f^{n}, n \in \mathbf{N}$, the $n$th iterate of $f$. The Fatou set, $F(f)$, is defined to be the set of points, $z \in \mathbf{C}$, such that $\left(f^{n}\right)_{n \in \mathbf{N}}$ forms a normal family in some neighbourhood of $z$. The complement, $J(f)$, of $F(f)$ is called the Julia set of $f$. An introduction to the basic properties of these sets can be found in, for example, 3 .

The first results on the iteration of transcendental entire functions were given by Fatou in [8. There are several general questions in [8], most of which have now been solved. One question that remains open is based on the following observation of Fatou: for the functions $f(z)=z+1+e^{-z}$ and $f(z)=h \sin z$ (where $0<h<1$ ), there are infinitely many unbounded curves $\gamma_{k}, k \in \mathbf{Z}$, with the property that, for $z \in \gamma_{k}$, we have $f^{n}(z) \rightarrow \infty$ as $n \rightarrow \infty$. Fatou asked whether this property holds more generally. It is known that this property does hold for certain families of functions (see [6] and [12]), but it is still not known whether this property holds in general.

This question of Fatou concerns the so-called 'escaping set'

$$
I(f)=\left\{z: f^{n}(z) \rightarrow \infty \text { as } n \rightarrow \infty\right\},
$$

which was first studied for a general transcendental entire function $f$ by Eremenko 7]. He proved that

$$
\begin{gathered}
I(f) \neq \emptyset, \\
J(f)=\partial I(f), \\
I(f) \cap J(f) \neq \emptyset,
\end{gathered}
$$

$\overline{I(f)}$ has no bounded components.

Received by the editors April 4, 2003 and, in revised form, November 28, 2003. 2000 Mathematics Subject Classification. Primary 37F10; Secondary 37F45.

(C)2004 American Mathematical Society Reverts to public domain 28 years from publication 
Eremenko conjectured that it may be possible to replace $\overline{I(f)}$ with $I(f)$ in (1.4), a problem that still remains open; see [12] for a proof in the case of exponential maps.

In this paper we prove results that give partial answers to both Fatou's and Eremenko's questions concerning the set $I(f)$. In order to do this we consider the following subset of $I(f)$, which was introduced by Bergweiler and Hinkkanen in [5]:

$$
A(f)=\left\{z: \text { there exists } L \in \mathbf{N} \text { such that }\left|f^{n}(z)\right|>M\left(R, f^{n-L}\right) \text {, for } n>L\right\} .
$$

Here, $M(r, f)=\max _{|z|=r}|f(z)|$ and $R$ can be taken to be any value such that $R>\min _{z \in J(f)}|z|$. Properties (1.1), (1.2) and (1.3) also hold for $A(f)$ (see [5] and the remarks at the end of this paper), and both $A(f)$ and $I(f)$ are completely invariant.

Our main result is the following.

Theorem 1. Let $f$ be a transcendental entire function. Then each $z_{0} \in A(f)$ lies in an unbounded closed connected subset of $A(f)$; in particular, $A(f)$ has no bounded components.

This goes some way to answering Fatou's question as it shows that $I(f)$ has at least one unbounded component. It also goes some way to answering Eremenko's question as it shows that $\overline{I(f)}$ can be replaced with $A(f)$ in (1.4).

When $F(f)$ has a multiply connected component, we can show much more than this; here, $I(f)$ has exactly one unbounded component and no bounded components, showing that Eremenko's conjecture is true in this case.

Theorem 2. Let $f$ be a transcendental entire function and suppose that $F(f)$ has a multiply connected component. Then

(a) $A(f)$ is connected and unbounded and contains the closure of every multiply connected component of $F(f)$;

(b) $I(f)$ is connected and unbounded.

Baker [1] showed that it is possible for $F(f)$ to have a multiply connected component $U$. He also showed that $U \subset I(f)$ and, for $n \geq n_{0}$, the sets $f^{n}(U)$ lie in bounded multiply connected components $U_{n}$ of $F(f)$ surrounding 0 , with $U_{n} \rightarrow \infty$; see [2 Theorem 3.1]. For this reason, in [13, we introduced the name Baker wandering domain for such a component of $F(f)$.

\section{Basic Properties of $A(f)$}

In this section we prove a number of basic properties of $A(f)$ including, for completeness, some that were stated but not proved in $[5$. Several of these properties are used in the proofs of Theorems 1 and 2. To do this, it is helpful to introduce the following alternative definition of $A(f)$ :

$$
B(f)=\left\{z \text { : there exists } L \in \mathbf{N} \text { such that } f^{n+L}(z) \notin \widetilde{f^{n}(D)} \text {, for } n \in \mathbf{N}\right\},
$$

where $D$ is any open disc meeting $J(f)$ and $\widetilde{U}$ denotes the union of $U$ and its bounded complementary components. We prove several basic properties of the set $B(f)$ and then show that $B(f)=A(f)$. 
In what follows we often use the property that if $G$ is a bounded region, then

$$
f(\widetilde{G}) \subset \widetilde{f(G)}
$$

which holds because if $\gamma$ is any simple closed curve in $G$, then the image under $f$ of the inside of $\gamma$ lies inside $f(\gamma)$, and hence in $\widetilde{f(G)}$.

Lemma 2.1. The set $B(f)$ has the following properties:

(a) $B(f)$ is independent of $D$;

(b) $B(f)$ is completely invariant;

(c) $B\left(f^{p}\right)=B(f)$, for $p \in \mathbf{N}$;

(d) if $g=h^{-1} f h$, where $h(z)=a z+b, a \neq 0$, then $B(f)=h(B(g))$.

Proof. Properties (a), (b) and (c) follow easily from the blowing-up property of $J(f)$. We prove just property $(\mathrm{c})$.

Let $D$ be an open disc meeting $J(f)$. If $z \in B(f)$, then there exists $L \in \mathbf{N}$ such that

$$
f^{n+L}(z) \notin \widehat{f^{n}(D)} \text {, for } n \in \mathbf{N} \text {. }
$$

By the blowing-up property, $\widetilde{f^{K}(D)} \supset D$ for all large $K$. So $\widetilde{f^{n+K}(D)} \supset \widetilde{f^{n}(D)}$, for $n \in \mathbf{N}$, by (2.1), and hence

$$
f^{p n+L+K}(z) \notin \widehat{f^{p n}(D)} \text {, for } n \in \mathbf{N} \text {. }
$$

Since it is possible to choose $K$ such that $p$ divides $L+K$, it follows that $z \in B\left(f^{p}\right)$.

On the other hand, if $z \in B\left(f^{p}\right)$, then there exists $l \in \mathbf{N}$ such that

$$
\left(f^{p}\right)^{n+l}(z) \notin \widehat{\left(f^{p}\right)^{n}(D)} \text {, for } n \in \mathbf{N} \text {. }
$$

Thus, by (2.1),

$$
f^{p n+p l-k}(z) \notin \widehat{f^{p n-k}(D)}, \text { for } n \in \mathbf{N}, k=1,2, \cdots, p-1,
$$

and so $z \in B(f)$.

Property (d) is true because the corresponding orbits, discs, image sets and Julia sets are congruent to each other under $h$.

To prove that $B(f)=A(f)$, we use a generalisation of Bohr's theorem 9, page 170]. We write $B(0, R)=\{z:|z|<R\}$ and $\bar{B}(0, R)=\{z:|z| \leq R\}$.

Lemma 2.2. Let $f$ be entire with a 2-cycle $z_{1}, z_{2}$ (possibly coincident). There is an absolute constant $c, 0<c<1$, such that if

$$
\left|z_{1}\right|<2 R / 5 \text { and }\left|z_{2}\right|<c M(R / 2, f)
$$

then

$$
f(B(0, R)) \supset\left\{w:|w|=r^{\prime}\right\}, \text { where } r^{\prime} \geq c M(R / 2, f) .
$$

Proof. Suppose that $f$ omits in $B(0, R)$ two values $w_{1}$ and $w_{2}$ such that

$$
\left|w_{1}\right|=c M(R / 2, f) \quad \text { and } \quad\left|w_{2}\right|=2 c M(R / 2, f),
$$

where $c$ has yet to be chosen. Put

$$
\phi(z)=\frac{f(\mu(z))-w_{1}}{w_{2}-w_{1}}, \quad \text { where } \mu(z)=\frac{R^{2}\left(z+z_{1}\right)}{R^{2}+\overline{z_{1}} z} .
$$


Then $\mu$ maps $B(0, R)$ onto itself and

$$
|\phi(0)|=\left|\frac{z_{2}-w_{1}}{w_{2}-w_{1}}\right| \leq \frac{2 c M(R / 2, f)}{c M(R / 2, f)}=2 .
$$

Also, $\phi$ omits 0 and 1 in $B(0, R)$, so Schottky's theorem [9] page 169], applied to the function $\phi(R t),|t|<1$, gives

$$
\log |\phi(z)| \leq\left(\log ^{+}|\phi(0)|+C\right)\left(\frac{1+|z| / R}{1-|z| / R}\right), \quad \text { for }|z|<R,
$$

where $C$ is an absolute positive constant. Thus, if $z \in \bar{B}(0,3 R / 4)$, then we have $|\phi(z)| \leq A=\exp (7(\log 2+C))$ and so, by $(2.2)$,

$$
\begin{aligned}
|f(\mu(z))| & \leq\left|w_{1}\right|+\left|w_{2}-w_{1}\right||\phi(z)| \\
& \leq c(1+3 A) M(R / 2, f) \\
& <M(R / 2, f),
\end{aligned}
$$

provided that $c<(1+3 A)^{-1}$. But this contradicts the definition of $M(R / 2, f)$ because $\left|z_{1}\right|<2 R / 5$ implies that $\mu(\bar{B}(0,3 R / 4)) \supset \bar{B}(0, R / 2)$. Therefore, if $c<$ $(1+3 A)^{-1}$, then $f$ cannot omit two values satisfying $(2.2)$. The lemma follows.

We also use the following variant of Schwarz's lemma.

Lemma 2.3. Let $f$ be entire with a 2-cycle $z_{1}, z_{2}$ (possibly coincident), and let $C$ be an arbitrary constant, $0<C<1$. If

$$
\left|z_{1}\right|<C R / 8 \text { and }\left|z_{2}\right|<C M(R, f) / 8,
$$

then

$$
M(C R / 8, f) \leq C M(R, f) .
$$

Proof. Let $d\left(z, z^{\prime} ; G\right)$ denote the hyperbolic metric in a domain $G$. In particular,

$$
d(z, 0 ; B(0, R))=\log \left(\frac{1+|z| / R}{1-|z| / R}\right),
$$

or, equivalently, $|z| / R=\tanh \left(\frac{1}{2} d(z, 0 ; B(0, R))\right)$. Then, by the contraction property of the hyperbolic metric, we have, for $z \in \bar{B}(0, C R / 8)$,

$$
\begin{aligned}
d\left(f(z), z_{2} ; B(0, M(R, f))\right) & \leq d\left(f(z), z_{2} ; f(B(0, R))\right) \\
& \leq d\left(z, z_{1} ; B(0, R)\right) \\
& \leq d(z, 0 ; B(0, R))+d\left(z_{1}, 0 ; B(0, R)\right) \\
& \leq 2 \log \left(\frac{1+C / 8}{1-C / 8}\right) \leq C .
\end{aligned}
$$

Hence, for $z \in \bar{B}(0, C R / 8)$,

$$
\begin{aligned}
d(f(z), 0 ; B(0, M(R, f))) & \leq C+d\left(z_{2}, 0 ; B(0, M(R, f))\right) \\
& \leq C+\log \left(\frac{1+C / 8}{1-C / 8}\right) \leq 2 C
\end{aligned}
$$

so

$$
|f(z)| \leq M(R, f) \tanh C \leq C M(R, f),
$$

as required. 
Lemma 2.4. $B(f)=A(f)$.

Proof. Clearly $A(f) \subset B(f)$. To prove the reverse inclusion, we use the fact that $f$ is known to have a 2 -cycle $z_{1}, z_{2}$ which must also be a 2-cycle (possibly coincident) of $f^{n}$, for each $n \in \mathbf{N}$. We now choose $R>0$ such that

$$
\begin{gathered}
B(0, c R / 16) \cap J(f) \neq \emptyset, \\
\left|z_{i}\right|<c R / 16, \text { for } i=1,2,
\end{gathered}
$$

and

$$
\left|z_{i}\right|<c M\left(R / 2, f^{n}\right) / 8, \text { for } i=1,2, n \in \mathbf{N},
$$

where $c$ is the constant in Lemma 2.2. Note that (2.5) can be satisfied because $M\left(R / 2, f^{n}\right) \rightarrow \infty$ as $n \rightarrow \infty$, by (2.3) and the blowing-up property of $J(f)$.

Now put $D=B(0, R)$. If $z \in B(f)$, then it follows from (2.3) that there exists $L \in \mathbf{N}$ such that

$$
f^{n+L}(z) \notin \widetilde{f^{n}(D)} \text {, for } n \in \mathbf{N} .
$$

The hypotheses of Lemma 2.2 are satisfied for the functions $f^{n}, n \in \mathbf{N}$, by (2.4) and (2.5), and so

$$
f^{n}(D) \supset\left\{w:|w|=r_{n}\right\}, \text { where } r_{n} \geq c M\left(R / 2, f^{n}\right), \text { for } n \in \mathbf{N} .
$$

Thus

$$
\left|f^{n+L}(z)\right|>c M\left(R / 2, f^{n}\right), \text { for } n \in \mathbf{N} .
$$

The hypotheses of Lemma 2.3 are satisfied for the functions $f^{n}, n \in \mathbf{N}$, with $C=c$ and $R$ replaced by $R / 2$ (by (2.4) and (2.5)), and so

$$
\left|f^{n+L}(z)\right|>c M\left(R / 2, f^{n}\right)>M\left(c R / 16, f^{n}\right) \text {, for } n \in \mathbf{N} .
$$

Thus $z \in A(f)$, by (2.3).

\section{Proof of Theorem 1}

We first choose $R>0$ so that

$$
B(0, R) \cap J(f) \neq \emptyset .
$$

Then put

$$
D_{n}=f^{n}(B(0, R)), \text { for } n=0,1,2, \ldots,
$$

and let $E_{n}$ be the complement of $\widetilde{D_{n}}$.

Now suppose that $z_{0}$ satisfies

$$
f^{n}\left(z_{0}\right) \in E_{n}, \text { for } n \in \mathbf{N} .
$$

Let $L_{n}$ be the component of $f^{-n}\left(E_{n}\right)$ which contains $z_{0}$. Then $L_{n}$ is closed and also unbounded, since $f^{n}$ is analytic. Moreover,

$$
L_{n} \supset L_{n+1} \text {, for } n \in \mathbf{N} \text {. }
$$

Indeed, if $L_{n+1} \backslash L_{n} \neq \emptyset$, then (because $L_{n+1} \cap L_{n} \neq \emptyset$ ) we can choose $z^{\prime}$ in $L_{n+1}$ so that $f^{n}\left(z^{\prime}\right) \notin E_{n}$. Hence $f^{n+1}\left(z^{\prime}\right) \notin E_{n+1}$, by (2.1), a contradiction.

Now, for $n \in \mathbf{N}$,

$$
K_{n}=L_{n} \cup\{\infty\}
$$


is a closed connected subset of $\hat{\mathbf{C}}$. It follows from (3.2) that $K_{n} \supset K_{n+1}$, for $n \in \mathbf{N}$, and so

$$
K=\bigcap_{n=1}^{\infty} K_{n}
$$

is also a closed connected subset of $\hat{\mathbf{C}}$, which contains $z_{0}$ and $\infty$. Let $\Gamma$ be the component of $K \backslash\{\infty\}$ which contains $z_{0}$. Then $\Gamma$ is closed in $\mathbf{C}$ and unbounded; see [11 page 84].

We claim that $\Gamma \subset A(f)$. For if $z \in \Gamma$, then $f^{n}(z) \in E_{n}$, for $n \in \mathbf{N}$, and so

$$
f^{n}(z) \notin \widetilde{D_{n}}=\widetilde{f^{n}\left(D_{0}\right)}, \text { for } n \in \mathbf{N} .
$$

Thus $z \in B(f)$, by (3.1), so $z \in A(f)$, by Lemma 2.4 .

Now suppose that $z^{\prime} \in A(f)=B(f)$. Then, by (3.1) and the definition of $B(f)$, there is a positive integer $L=L\left(z^{\prime}\right)$ such that

$$
f^{n+L}\left(z^{\prime}\right) \notin \widetilde{D_{n}}, \text { for } n \in \mathbf{N} .
$$

Thus $z^{\prime \prime}=f^{L}\left(z^{\prime}\right)$ satisfies

$$
f^{n}\left(z^{\prime \prime}\right) \notin \widetilde{D_{n}} \text {, for } n \in \mathbf{N} \text {. }
$$

By the previous argument, $z^{\prime \prime}$ lies in an unbounded closed connected subset, $\Gamma^{\prime \prime}$ say, of $A(f)$. Let $\Gamma^{\prime}$ denote the component of $f^{-L}\left(\Gamma^{\prime \prime}\right)$ which contains $z^{\prime}$. Then $\Gamma^{\prime}$ is closed and also unbounded, since $f$ is analytic. Since $A(f)$ is completely invariant, by Lemma 2.1(b) and Lemma 2.4, we deduce that $\Gamma^{\prime} \subset A(f)$. Thus the proof of Theorem 1 is complete. (Note that if $\Gamma^{\prime}$ is constructed in this way, then by (3.3) each $z \in \Gamma^{\prime}$ satisfies (3.4).)

\section{Proof of Theorem 2}

As in the proof of Theorem 1, we choose $R>0$ so that

$$
B(0, R) \cap J(f) \neq \emptyset
$$

and put

$$
D_{n}=f^{n}(B(0, R)), \text { for } n=0,1,2, \ldots
$$

Now let $U_{0}$ be a Baker wandering domain, that is, a multiply connected component of $F(f)$. As shown by Baker [2, Theorem 3.1], the sets $f^{n}\left(U_{0}\right), n=1,2, \ldots$, lie in bounded multiply connected components $U_{n}$ of $F(f)$ and $U_{n} \rightarrow \infty$ as $n \rightarrow \infty$ in such a way that, for $n \geq N$ say, we have $U_{n} \subset \widetilde{U_{n+1}}$. We can assume that $D_{0} \subset \widetilde{U_{N}}$.

Now $f^{n}\left(\widetilde{U_{N}}\right) \subset \widetilde{U_{N+n}}$, by $(2.1)$. Thus

$$
D_{n}=f^{n}\left(D_{0}\right) \subset \widetilde{U_{N+n}} \text {, for } n \in \mathbf{N} .
$$

Now suppose that $z \in \bar{U}_{N}$. Then $f^{n+1}(z) \in \bar{U}_{N+n+1}$, and so $f^{n+1}(z) \notin \widetilde{U_{N+n}}$, for $n \in \mathbf{N}$. Thus, by (4.1) and (4.2), we deduce that $z \in B(f)=A(f)$. Hence $\bar{U}_{N} \subset A(f)$, and so $\bar{U}_{0} \subset A(f)$, by the complete invariance of $A(f)$; see Lemma 2.1(b) and Lemma 2.4.

Thus the closure of any Baker wandering domain lies in $A(f)$. Since any unbounded connected set must meet $U_{n}$ for large $n$, we deduce from Theorem 1 that $A(f)$ is connected. This proves part (a). 
In [5, Lemma 3] it was proved that $J(f) \subset \overline{A(f)}$. Hence

$$
A(f) \cup(I(f) \cap J(f)) \subset \overline{A(f)},
$$

and so $A(f) \cup(I(f) \cap J(f))$ is a connected subset of $I(f)$.

If $z \in I(f) \cap F(f)$, then $z$ belongs to a Baker domain or a wandering domain, by the classification of components of $F(f)$; see, for example, [3]. Baker domains are unbounded and so cannot exist in this case as they would meet the Baker wandering domains. Similarly, there can be no unbounded wandering domains and so it remains to consider any bounded wandering domains in $I(f)$. Let $V_{0}$ be such a wandering domain and put $V_{n}=f^{n}\left(V_{0}\right)$. Since $V_{0} \subset I(f)$, it follows that, for $n \geq N_{1}$ say,

$$
V_{n} \subset \mathbf{C} \backslash \widetilde{U_{m(n)}},
$$

where $m(n) \rightarrow \infty$ as $n \rightarrow \infty$. Thus

$$
f^{n}\left(\bar{V}_{0}\right) \subset \bar{V}_{n} \subset \mathbf{C} \backslash \widetilde{U_{m(n)}}, \text { for } n \geq N_{1},
$$

and so $\partial V_{0} \subset I(f) \cap J(f)$. Hence $V_{0}$ lies in the same component of $I(f)$ as does the connected set $A(f) \cup(I(f) \cap J(f))$. Part (b) now follows.

\section{REMARKS}

1. In [5] the proof was given that, for a transcendental entire function $f$, we have $J(f) \subset \overline{A(f)}$. It follows that $J(f) \subset \partial A(f)$, and it was stated in 5 that $J(f)=\partial A(f)$. We outline a proof of the inclusion $\partial A(f) \subset J(f)$, based on our Theorem 2. If this inclusion is not true, then there must be a closed disc $\Delta_{0}$ in $F(f)$ which meets both $A(f)$, at $z$ say, and the complement of $A(f)$, at $z^{\prime}$ say. It follows from Theorem 2 that $\Delta_{0}$ cannot lie in a multiply connected component of $F(f)$. If $\Delta_{0}$ lies in a simply connected component of $F(f)$, then it follows from [13. Theorem 3(b)], for example, that there exists $C, 0<C<1$, such that

$$
\left|f^{n}\left(z^{\prime}\right)\right| \geq C\left|f^{n}(z)\right| \text {, for } n \in \mathbf{N} .
$$

Thus, since $z \in A(f)$, there exist $R>0$ and $L \in \mathbf{N}$ such that $J(f) \cap B(0, C R / 8) \neq \emptyset$ and

$$
\left|f^{n+L}\left(z^{\prime}\right)\right| \geq C\left|f^{n+L}(z)\right| \geq C M\left(R, f^{n}\right) \geq M\left(C R / 8, f^{n}\right) \text {, for } n \in \mathbf{N},
$$

by Lemma 2.3, together with the justification of (2.5). Hence $z^{\prime} \in A(f)$, which is a contradiction.

2. We also observe that property (1.3) holds with $I(f)$ replaced by $A(f)$. It follows from Theorem 2 that $A(f) \cap J(f) \neq \emptyset$ if $f$ has a Baker wandering domain. It is also true that $A(f) \cap J(f) \neq \emptyset$ if $f$ has no Baker wandering domain. Indeed, Eremenko's proof of property (1.1) gives a point $z_{0}$ such that

$$
\left|f^{n+1}\left(z_{0}\right)\right| \geq \frac{1}{2} M\left(\left|f^{n}\left(z_{0}\right)\right|, f\right) \quad \text { as } n \rightarrow \infty,
$$

and also, assuming the absence of Baker wandering domains, such that $z_{0} \in J(f)$ [7, Theorems 1 and 2]. Hence $z_{0} \in A(f) \cap J(f)$, by [5, Lemma 2]. This result of Eremenko shows that if $f$ has no Baker wandering domains, then $J(f)$ has a dense subset of points $z_{0}$ such that (4.3) holds.

3. We do not know an example of a transcendental entire function which has a Baker wandering domain and also a (bounded) simply connected wandering domain, 
and it is natural to ask whether this can occur. It is possible for an entire function to have a bounded, simply connected, wandering domain. For example, the function

$$
f(z)=2-\log 2+2 z-e^{z}
$$

discussed by Bergweiler in [4, has a Fatou component containing the point $\log 2+$ $2 \pi i$, which is simply connected and wandering [4, Section 2]. This component can also be shown to be bounded (by finding a Jordan curve around $\log 2+2 \pi i$, which is mapped outside itself by $f$; see [10]).

4. The reasoning in Remark 1 shows that any simply connected component of $F(f)$ which meets $A(f)$ must lie entirely in $A(f)$. We do not know of a case where such a simply connected component of $F(f)$ exists.

\section{REFERENCES}

1. I.N. Baker. An entire function which has wandering domains. J. Austral. Math. Soc. Ser. A 22 (1976), 173-176. MR0419759 (54:7777)

2. I.N. Baker. Wandering domains in the iteration of entire functions. Proc. London Math. Soc. (3) 49 (1984), 563-576. MR0759304 (86d:58066)

3. W. Bergweiler. Iteration of meromorphic functions. Bull. Amer. Math. Soc., 29 (1993), 151188. MR1216719 (94c:30033)

4. W. Bergweiler. Invariant domains and singularities. Math. Proc. Camb. Phil. Soc., 117 (1995), 525-532. MR 1317494 (96b:30055)

5. W. Bergweiler and A. Hinkkanen. On semiconjugation of entire functions. Math. Proc. Camb. Phil. Soc., 126 (1999), 565-574. MR1684251 (2000c:37057)

6. R.L. Devaney and F. Tangerman. Dynamics of entire functions near the essential singularity. Ergod. Th. and Dynam. Sys., 6 (1986), 489-503. MR0873428 (88e:58057)

7. A.E. Eremenko. On the iteration of entire functions. Dynamical systems and ergodic theory, Banach Center Publ. 23 (Polish Scientific Publishers, Warsaw, 1989) 339-345. MR1102727 (92c:30027)

8. P. Fatou. Sur l'itération des fonctions transcendantes entières. Acta Math., 47 (1926), 337-370.

9. W.K Hayman. Meromorphic functions. Clarendon Press, Oxford, 1964. MR.0164038 (29:1337)

10. M. Kisaka, On the connectivity of Julia sets of transcendental entire functions. Ergodic Theory Dynam. Systems, 18 (1998), 189-205. MR1609471 (99a:30033)

11. M.H.A. Newman. Elements of the topology of plane sets of points, Cambridge University Press, 1961. MR0132534 (24A:2374)

12. D. Schleicher and J. Zimmer. Escaping points of exponential maps. J. London Math. Soc. (2), 67 (2003), 380-400. MF.1956142 (2003k:37067)

13. P.J. Rippon and G.M. Stallard. On sets where iterates of a meromorphic function zip towards infinity. Bull. London Math. Soc., 32 (2000), 528-536. MR.1767705 (2001g:30019)

Department of Pure Mathematics, The Open University, Walton Hall, Milton Keynes MK7 6AA, United KingDOM

E-mail address: p.j.ripponœopen.ac.uk

Department of Pure Mathematics, The Open University, Walton Hall, Milton Keynes MK7 6AA, United Kingdom

E-mail address: g.m.stallard@open.ac.uk 